\title{
ISO 9002 NO CENTRO DE TERAPIA INTENSIVA PEDIÁTRICO DO HOSPITAL ISRAELITA ALBERT EINSTEIN
}

\author{
ISO 9002 IN PEDIATRIC INTENSIVE THERAPY CENTER OF "HOSPITAL \\ ISRAELITA ALBERT EINSTEIN" \\ ISO 9002 EN EL CENTRO DE TERAPIA INTENSIVA PEDIÁTRICO DEL \\ HOSPITAL ISRAELITA ALBERT EINSTEIN
}

\author{
Denise Pourrat Dal Gé Lacerda' \\ Mariana Lucas Rocha ${ }^{2}$ \\ Regiane Pereira Santos ${ }^{3}$
}

\begin{abstract}
RESUMO: A implantação do programa de qualidade no CTI-P, do HIAE, iniciou em Jan/96, visando melhoria continua da assistência à criança e familia e o desenvolvimento profissional. Utilizamos como ferramenta a Norma ISO $9002 / 94$, que necessitou ser interpretada para a realidade hospitalar. Definimos o cronograma de trabalho, a politica de qualidade norteada pela missão da Instituição e consolidada na finalidade de trabalho do serviço. Atendendo aos vinte itens da Norma, procedemos a documentaçāo, através da elaboraçāo do Manual da Qualidade, da revisão e padronização de rotinas e procedimentos. A equipe multiprofissional foi sensibilizada e envolvida a participar de todo o processo de implantaçăo e avaliação do programa de Qualidade. Em Mar/98, após auditoria pela British Standards Institution foi conferido ao CTI-P - HIAE certificado ISO 9002. Ressaltamos a importåncia do papel do enfermeiro como líder em todo este processo. Cabe à equipe o desafio permanente da manutenção do programa de qualidade.
\end{abstract}

PALAVRAS-CHAVE: qualidade, centro de terapia intensiva, pediatria, ISO 9002

\section{INTRODUÇÃO}

O Centro de Terapia Intensiva Pediátrico (CTI-P) do Hospital Israelita Albert Einstein (HIAE) possui 4 leitos de terapia intensiva e 4 leitos de semi-intensiva, e dá retaguarda a população pediátrica proveniente da enfermaria assistencial, ambulatório pediátrico, posto de atendimento da comunidade, unidade de pediatria particular e outras unidades do HIAE, pronto atendimento, consultórios médicos, serviço de apoio e diagnóstico e centro cirúrgico.

Desde recém-nascido externo a instituição, à adolescentes até 16 anos. O quadro de funcionários é composto pelo médico chefe, enfermeira chefe, enfermeira epidemiológica, um médico diarista, um médico plantonista ( no total de 12 médicos), uma enfermeira e 4 auxiliares em cada periodo, fisioterapeutas, nutricionista e auxiliar administrativo.

Frente às mudanças nas expectativas dos clientes, no papel dos cuidadores, no avanço da tecnologia e também por questões econômicas sentimos a necessidade de reestruturar o serviço

\footnotetext{
' Enfermeira encarregada do Centro de Terapia Intensiva (CTI-P), Pediatria Particular e Assistencial, e Ambulatório de Pediatria do Hospital Israelita Albert Einstein (HIAE)

${ }^{2}$ Enfermeira assistencial do CTI-P/HIAE e Pediatria Particular e Assistencial, Mestranda da EEUSP. Residência em Enfermagem Pediatrica.

${ }^{3}$ Enfermeira Epidemiológica e RA do CTI-P/HIAE. Especializada em Administração Hospitalar. Residência Geral.
} 
oferecido aos nossos pacientes, embasando nossas metas na garantia da qualidade da assistência prestada.

Sabemos que a preocupação com a qualidade surgiu nos ramos das indústrias com o advento da segunda guerra mundial. Há algum tempo atrás a preocupação com a qualidade dos produtos não era muito evidente, os poucos fabricantes existentes na época tinham todo os seus produtos consumidos pelo próprio mercado interno, e se alguma parte sobrava era comercializada para aqueles que ainda estavam em desenvolvimento e não produziam nada industrializado. Atualmente o mercado está cada vez mais competitivo e o produtor não pode mais ficar voltado apenas para a produção em quantidade, muitos estão produzindo e o que se espera é um produto barato e de qualidade.

Vários trabalhos realizados dentro dos principios da qualidade tiveram suas bases sedimentadas nas teorias de Crosby e Deming. Crosby citado por Ishikawa (1993) defende a idéia que o produto deve atender a necessidade do cliente e reconhece a importância da relação custo-qualidade, foi Crosby que introduziu na indústria o conceito "defeito zero"e a necessidade de se fazer corretamente da primeira vez. Deming (apud Ishikawa, 1993) auxiliou na recuperação da economia japonesa com a estratégia de continuo aprimoramento para obtenção da qualidade.

Qualidade tornou-se um termo bastante empregado em qualquer ramo de negócio e, por isso, desgastado. Para Puri (1994): "é um conceito multifacetado e complexo. É a soma de todas as caracteristicas de um produto ou serviço que contribuem para sua superioridade ou excelência.". Ferreira (1986) diz: "Qualidade é a propriedade ou atributo ou condição das coisas ou das pessoas, capaz de distingui-las das outras e de deterninar a natureza."

Qualquer pessoa hoje em dia já teria um conceito de qualidade sobre determinado produto, seja porque este produto atende as características esperadas por esse cliente, seja porque tem um preço que a pessoa considera acessivel, seja porque é fácil de se obter, etc... E este é um dos fatores que gera sinônimos incorretos ou até mesmo uso indevido desta palavra.

Ramos et al. (1994) cita que: "A Joint Comission of Acreditation Healthcare Organizations (JCAHO) define Qualidade como o grau de cuidado prestado ao paciente que aumenta a probabilidade de obter o resultado desejado e diminui a probabilidade de resultados indesejados, oferecido de acordo com o estado atual de conhecimentos."

Segundo Vaz (1997), citando Moller a palavra qualidade pode ser usada de várias formas e com vários significados, sendo dificil descrever de forma clara e objetiva. É influenciada por fatores como cultura, experiência, idade, formação, necessidade numa dada situação, humor e atividade; e todos conseguem de uma forma generalizada categorizar um serviço ou produto como sendo de boa ou má qualidade, se satifaz ou não as nossas necessidades.

É Donabedian , citado por Berwick et al. (1994), considerado o fundador do campo da garantia da qualidade nos serviços de saúde, e define os alvos da avaliação: estruturas são os recursos que estão disponiveis para se oferecer o serviço; processo é o próprio atendimento; e resultados são as consequências avaliadas do atendimento. Dentro da área de saúde o termo Qualidade vem sendo empregado nos últimos anos com bastante intensidade. Alguns autores como Ramos et al. (1994) comentam que há uma controvérsia na questão do embasamento da qualidade nas expectativas do cliente na referida área, já que o paciente pode ser capaz de avaliar os serviços de hotelaria e menos capaz de avaliar os serviços de saúde.

Para Juran (citado por Berwick et al. 1994): "Na qualidade dos serviços de saúde ouvir a voz do cliente inclui a avaliação sistemática tanto das atitudes dos pacientes como do impacto dos processos de tratamento sobre a saúde dos pacientes."

A preocupação com o tema qualidade na assistência à saúde iniciou de forma sistematizada no Hospital Israelita Albert Einstein em 1989, com a formação do Sistema Einstein de Qualidade, com os Indicadores de Qualidade de Enfermagem, e a notificação e análise de Ocorrências Adversas ao Paciente. Com a supervisão de uma empresa de consultoria externa fez-se um diagnóstico da situação do hospital, em 1995, e propôs-se a implantação de um 
programa de qualidade em 4 setores do hospital: Banco de sangue, Laboratório de Análises Clínicas, Centro de Terapia Intensiva Adulto ou Centro de Terapia Intensiva Pediátrico. Formalizando o inicio do planejamento de um programa de qualidade de forma sistematizada.

O Sistema da Qualidade do CTI-P foi concebido tomando-se por base os principios e elementos operacionais constantes da Norma NBR ISO 9002:1994. Estas normas foram adaptadas aos serviços de saúde, uma que vez que foram elaboradas para uso nas indústrias. Foram utilizados os manuais da qualidade elaborados pelo Laboratório de Análises Clinicas e Centro de Terapia Intensiva Adulto que naquela época já haviam iniciado o processo de implantação do mesmo programa de qualidade. Tendo como comprometimento da administração o envolvimento do Comitê Tático de Administração e as Gerências Médica e de Enfermagem.

Neste momento do inicio da implantação do Programa de Qualidade, um marco bastante importante foi o envolvimento de toda a equipe neste projeto, e a conscientização de uma politica de qualidade que retratasse a que se destinava o CTI-P, com o compromisso de atender as expectativas do cliente, padronizar procedimentos e oferecer um atendimento com excelência e qualidade. A participação efetiva dos membros que compõem a equipe multiprofissional é sem dúvida um elemento chave para o sucesso da implantação do programa de qualidade, pois cada profissional deve estar consciente da sua importância na melhoria da qualidade, aproveitando-se do potencial, da criatividade, sensibilidade e capacidade de cada um, no trabalho em equipe, uma vez que em todo o processo o trabalho em grupo é imprescindivel para a obtenção dos objetivos.

\section{OBJETIVO}

O objetivo deste trabalho é divulgar o processo de implantação do Programa de Qualidade ISO 9002 no Centro de Terapia Intensiva Pediátrico do Hospital Israelita Albert Eisntein, através do relato de experiência.

\section{SISTEMA DA QUALIDADE}

\section{ESTRUTURA ORGANIZACIONAL}

O planejamento do Programa de Qualidade iniciou em Novembro de 1995 com a formação do Grupo de Planejamento Estratégico (GPE) composto pela equipe multiprofissional: médico chefe, enfermeira chefe, fisioterapeuta chefe, enfermeiras, auxiliares de enfermagem, fisioterapeuta, auxiliar administratrivo, secretária e o Representante da Administração; tinha como responsabilidades definir e documentar a política da qualidade e gerenciar o programa de qualidade, implementar os grupos de trabalho para desenvolvimento de qualidade e pesquisa, gerenciar implemento de tecnologia e realizar periodicamente a análise do sistema de qualidade. O grupo passou a se reunir sistematicamente.

O Representante da Administração (RA), como preconizado pela norma NBR ISO 9002/ 94 , foi definido entre os profissionais do CTI-P, não podendo ser exercido pelo chefe. A função do RA é gerenciar o sistema da qualidade assegurando seu estabelecimento, sua implementação e manutenção de acordo com a norma; deve relatar, notificar e acompanhar as análises referentes às não conformidades e ocorrências adversas ao paciente e administrativas (OAP e OAA); coordenar e analisar auditorias internas para avaliar adequação e conformidade do sistema, verificar a implementação das ações definidas na análise crítica pela administração e acompanhar o trabalho dos grupos de assessoria e suas dificuldades, coordenar as revisões do Manual da Qualidade.

Apartir do Grupo de Planejamento Estratégico foram formados os Grupos de Assessoria, que são compostos pela equipe multiprofissional, e têm como objetivo dar subsidios técnicos, 
cientificos e de apoio ao GPE; além de terem autoridade e responsabilidade de desenvolver projetos relacionados ao gerenciamento, qualidade, pesquisa e ensino, sob supervisão e respaldo do GPE. Os grupos de assessoria são: Informática e documentação, Equipamentos, Processos, 5 Ss e Treinamento.

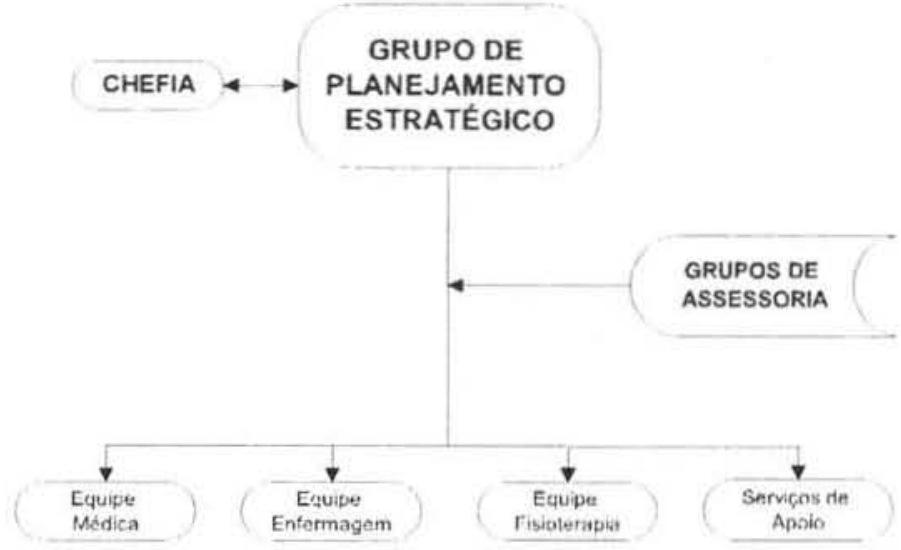

O sistema da qualidade do CTI-P foi estruturado e formalizado através de 3 niveis de documentação, com as respectivas atribuições:

Primeiro Nivel: Manual da Qualidade:

- descreve os principios básicos de qualidade: visão, finalidade, política, objetivos, organização, responsabilidade e recursos; e apresenta os procedimentos básicos que sào necessários para cumprir os requisitos na norma de referência adotada. É a linha mestra do sistema da qualidade.

Segundo Nivel: Manual de Rotinas e Fluxos:

- detalha as diversas rotinas e fluxos relacionados ao CTI-P, indicando como essas atividades são desempenhadas, de modo a implementar e manter os elementos do sistema da qualidade; e indica como, onde e quem desenvolve tais atividades.

Terceiro Nivel: Manual Técnico Operacional:

- detalha os diversos procedimentos e manuais operacionais relacionados ao CTI-P, indica como os procedimentos e manuais operacionais são implementados; indica como, onde e quem desenvolve tais atividades, incluindo requisitos básicos de qualificação profissional. Concomitante ao trabalho do GPE, os grupos de assessoria desenvolveram os manuais de Segundo e Terceiro Nivel, embasados já em documentos escritos em antigos manuais de rotinas e procedimentos já existentes nas unidades, reformulando-os, atualizando-os e adaptandoos conforme a Norma, e redigiram os manuais de equipamentos.

Após a elaboração destes manuais, os mesmos ficaram disponíveis de dois modos na unidade: em meio magnético através de um programa de software adquirido especificamente para este fim e em cópias de papel, de modo que todos os usuários pudessem ter acesso. Todos os profissionais que atuam no CTI-P têm acesso aos documento na versão ativa, necessários ao desempenho de suas funções, todos os documentos são analisados criticamente e aprovados pelo GPE. Documentos obsoletos são identificados como tal, sendo recolhidos dos pontos de emissão e uso, evitando sua utilização indevida (item 4.5 NBR ISO 9002/94).

\section{FINALIDADE, POLITICA DA QUALIDADE E VISÃO}

A Politica da Qualidade deve ser coerente com as metas organizacionais do fornecedor e as expectativas e necessidades de seus clientes. A politica e os objetivos qualidade são divulgados através do Manual da Qualidade, utilização de quadros de avisos, da educação 
contínua na forma de palestras, aulas ou treinamentos, de forma que todos tenham entendimento destes conceitos e das diretrizes do CTI Pediátrico.

Finalidade:

Prestar assistência médica à criança gravemente enferma, procurando reduzir a mortalidade, com menor número de complicações, de forma humanizada.

Politica da Qualidade:

A politica da Qualidade do CTI-P é caracterizada por: trabalhar em parceria com os médicos titulares; atender as necessidades e expectativas dos pacientes e seus familiares; ter uma postura profissional e ética; preocupar-se com a evolução profissional de seus familiares e com os recursos tecnológicos da unidade.

Visão:

Ser um centro de referência em qualidade médica, cientifica, tecnológica e humana em Terapia Intesiva Pediátrica.

Objetivos da qualidade:

- assegurar a qualidade de atendimento; controlar os indices de infecção; transmitir conhecimento científico; compromisso com a evolução da equipe multiprofissional; trabalhar em parceria com médicos titulares;

\section{RECURSOS}

O Grupo de Planejamento é responsável por identificar e providenciar recursos adequados para o desenvolvimento e implementação de atividades relacionadas ao Sistema da Qualidade dentro das disponibilidades e prioridades estabelecidas pela administração do HIAE.

A gestão dos recursos humanos, a fim de assegurar as condições de trabalho adequadas é analisada pleo Grupo de Planejamento em conjunto com as Gerências Médica e de Enfermagem e o Cômite Tático da Administração. Os recursos humanos são considerados elementos essenciais para a obtenção da Qualidade Total nos serviços. O comprometimento individual com os principios e objetivos da Qualidade é obtido através da educação e valorizaçăo do profissional. As chefia têm o compromisso de serem agentes facilitadores para que cada funcionário desenvolva seu potencial. As necessidades de treinamentos relativos às rotinas e aos procedimentos do Sistema da Qualidade são providenciadas de acordo com a rotina especifica, e se dá de forma sistemática e regular.

\section{TREINAMENTO}

O Grupo de Treinamento ficou responsável, juntamente com o Grupo de Planejamento Estratégico, de acompanhar o processo de implantação do Programa de Qualidade, elaborando treinamentos estratégicos com a finalidade de manter a equipe informada, motivada e atualizada.

Após o término da primeira versão do manual de primeiro nivel ou manual da qualidade, o treinamento iniciou com a apresentação pela alta administração do conteúdo relevante deste manual, por exemplo: a politica da qualidade, os valores e compromissos do CTI-P para os profissionais desta unidade. Este encontro propiciou discussões que permitiram trocas de informações e havia a preocupação de absorver sugestões provenientes da equipe multiprofissional que pudessem contribuir de forma positiva para as alterações finais do manual.

Com a preocupação dos manuais elaborados representarem o trabalho desenvolvido pela equipe do CTI-P e a instituição, o treinamento dos procedimentos, rotinas e equipamentos só foram concebidos após ampla revisão pela equipe de auxililiares de enfermagem, médicos, enfermeiras, fisioterapeutas e nutricionista os quais faziam parte dos grupos de assessoria. No total ocorreram 1.000 horas de treinamento, que aconteceram de maio de 96 a dezembro de 1997, no que se refere à equipe de enfermagem. O trabalho do grupo de treinamento é contínuo, 
implementando novas rotinas de trabalho quando necessário, como por exemplo: treinamento dos auditores internos da qualidade. A necessidade de treinamento pode ser detectada em quaisquer áreas e o fluxo para provimento de recursos se dá através do GPE. O grupo também elabora periodicamente um planejamento de treinamentos que contemplam temas básicos em terapia intensiva, como reanimação cardio-pulmonar cerebral.

\section{AQUISIÇÃO DE INSUMOSE FORNECEDORES}

Com a meta de garantir a qualidade dos produtos internos do HIAE foram confirmados contratos com áreas fornecedoras de serviços. Este procedimento está documentado de tal maneira que estabelece a responsabilidade e compromisso de ambas as partes, para manter a qualidade do produto fornecido. São considerados fornecedores internos: Serviços de Higiene, Serviço de Nutrição, Lavanderia, Almoxarifado, Serviços de Farmácia, Banco de Sangue, Laboratório de Análises Clínicas, Manutenção e Assistência Técnica, SAD, transporte interno de pacientes e Núcleo Técnico de Informática.

Insumos e materiais fornecidos pelo paciente/familiares, pertinentes ao tratamento e utilização durante a internação no CTI-P são checados antes da utilização quanto a qualidade, procedência, condições de armazenamento e validade, conforme rotina pré-estabelecida e registrado em prontuário.

\section{CONTROLE E TRATAMENTO DAS NÃO CONFORMIDADES}

As não conformidades referentes ao funcionamento do sistema da qualidade recebem tratamento adequado, impedindo a utilização de produto não conforme ou sendo submetido a análise critica para a melhoria da qualidade da unidade. Elas são detectados através de:

- reclamações dos clientes externos (pacientes, familiares, médico titular, convênios); etc...)

- reclamações dos clientes internos (médicos, enfermeiras, auxiliares, fisioterapeutas,

- avaliação da satisfação do cliente;

- auditorias internas e externas do Sistema da Qualidade;

- observação continuada dos processos pelos médicos, enfermeiras, fisioterapeutas e demais envolvidos no sistema.

As não conformidades são identificadas, documentadas, avaliadas, notificadas as áreas envolvidas, através das rotinas especificas. A autoridade para avaliar e fazer análise critica sobre as não conformidades é do RA.

\section{AÇÃO CORRETIVA E AÇÃO PREVENTIVA}

As ações corretivas têm como objetivo evitar a reincidência de problemas ocorridos ligados à qualidade do serviço prestado e as açōes preventivas evitar que problemas potenciais ocorram.

Os procedimentos, rotinas e macroprocessos do sistema da qualidade são passiveis de alteração em função de implementações de ações corretivas e preventivas. A implementação de Ações Corretivas leva em consideração: análise das reclamações dos clientes; análise crítca dos relatórios das Não-Conformidades, priorizando relação custo-benefício e os riscos envolvidos, e se serão eliminadas ou minimizadas; definição da melhor ação corretiva, transmissão de informações referentes às ações corretivas ao GPE, e o registro de todos os passos deste processo.

A implementação de Ações Preventivas inclui: uso adequado de fontes de informação sobre os processos de trabalho no CTI-P (procedimentos, registros, relatório de serviços associados, análise da causuística), para a detecção, análise e eliminação ou minimização de 
causas potenciais de problemas, definição e acompanhamento dos passos necessários para a implementação, a informação ao GPE e o registro de todo o processo.

\section{AUDITORIAS INTERNAS E EXTERNAS}

O manual da qualidade e o de rotinas descrevem para quê e como acontecem as auditorias internas do Sistema da Qualidade, e seu escopo. O planejamento é realizado formalmente. Os auditores internos são profissionais do CTI-P, treinados para esta função e atendem a um perfil de liderança pessoal, compreensão dos processos a serem auditados e intervenções positivas na abordagem com os auditados. As auditorias internas são realizadas conforme rotina préestabelecida e planejadas anualmente, acontecendo a cada 3 meses. Objetiva-se delinear a linha e o ritmo de trabalho, e acima de tudo ajustar o sistema detectando as não-conformidades e sugerindo planos de melhoria. Durante a auditoria externa do Sistema da Qualidade, que há a manutenção da certificação por orgão externo competente.

\section{ANÁLISE CRITICA PELA ADMINISTRAÇÃO}

O manual de primeiro nivel estabelece que periodicamente a alta administração faça uma avaliação geral do sistema da qualidade implantado, utilizando os relatórios das auditorias internas e externas, relatórios do serviço de atendimento ao Cliente e relatórios da "performance" dos fornecedores internos. Esta análise gera planos de ações e determina responsáveis e prazos para o cumprimento das tarefas, que serão avaliadas durante as reuniōes do GPE. Posteriormente esses planos são reavaliados pela reunião de análise critica seguinte.

\section{OPROCESSO DE CERTIFICAÇÃO}

No processo de certificação (propriamente dito) preocupou-se inicialmente em situar todos os profissionais do CTI-P em relação ao objetivos e diretrizes do Programa de Qualidade, na atualização das rotinas e procedimentos da unidade, e este período se encerrou com a primeira auditoria interna do sistema e a primeira reunião de análise critica, cumprindo todos os itens exigidos pela Norma ISO 9002.

A partir deste momento o Sistema da Qualidade do CTI-P do HIAE pode ser submetido a pré-auditoria de certificação, que aconteceu em Janeiro de 98 , realizada por uma instituição externa contratada: a BRITISH STANDARDS INSTITUTION (BSI). Na pré-auditoria o auditor faz o reconhecimento do sistema de qualidade implantado e o levantamento de não-conformidades que precisam ser ajustadas para a certificação, definindo então a data para a auditoria de certificação. Em março de 1998, após a auditoria externa pela BSI, o Centro de Terapia Intensiva Pediátrico do HIAE foi certificado segundo as Normas ISO 9002/94.

\section{CONSIDERAÇÖES FINAIS}

A manutenção do programa de qualidade é fundamentada no compromisso de trabalho da equipe e no enfoque dinâmico do processo.

O dinamismo do programa de qualidade se deve às avaliações periódicas por todos os profissionais envolvidos, clientes internos e externos e análise de năo-conformidades que geram as alterações no sistema em seus diversos niveis. É preciso que todos os profissionais tenham consciência que o grande desafio deste processo não é a conquista da certificação e, sim, a manutenção do programa, visando a finalidade, politica e objetivos propostos.

Para o cliente o programa de qualidade viabiliza um canal aberto de comunicação, resultando em melhoria continua com agilidade, e a garantia do compromisso da equipe em 
atender suas necessidades. Em relação à equipe o programa de qualidade oferece organização, referência no trabalho, educação continuada e aprimoramento profissional. Para a administração representa informações organizadas, processos definidos, marketing e economia hospitalar.

Devemos ressaltar a importância do papel do enfermeiro como líder no planejamento, implementação e manutenção do programa de qualidade. Servindo de elo entre o Comitê Administrativo, equipe hospitalar e cliente.

ABSTRACT: This study shows the process of implementation of a quality program in Pediatric Intensive Therapy Center of "Hospital Israelita Albert Einstein" which resulted in the certification of this service for the Standards ISO 9002/94. It points out the nurse's role as a leader in this process.

KEYWORDS: quality, intensive therapy center, pediatric

RESUMEN : La implantación del programa de calidad en el CTI-P, del HIAE, se inició en Enero/96 y su objetivo fue la mejoría continua de la asistencia al niño y familia y al desarrollo profesional. Utilizamos como herramienta la Norma ISO 9002/94 que necesitó ser interpretada dentro de la realidad hospitalaria. Definimos el cronograma de trabajo, la política de calidad orientada por la misión de la Institución y consolidada en la finalidad de trabajo del servicio. Atendiendo los veinte itens de la Norma, procedimos a la documentación, a través de la elaboración de un Manual de la Calidad y a la revisión y padronización de rutinas y procedimientos. El equipo multiprofesional fue sensibilizado y se les conllevó a participar de todo el proceso de implantación y evaluación del programa de Calidad. En Marzo/98 tras auditoria hecha por la British Standards Institution fue conferido al CTI-P HIAE el certificado ISO 9002. Resaltamos la importancia del papel del enfermero como líder durante todo el proceso. Toca al equipo el desafío permanente de mantener el programa de calidad.

PALABRAS CLAVE: calidad, centro de terapia intensiva, pediatria.

\section{REFERÊNCIAS BIBLIOGRÁFICAS}

BERWICK, D. M.; GODFREY, B; ROESSNER, J. Melhorando a qualidade dos serviços médicos, hospitalares e da saúde. São Paulo: Makron Books, 1994.

FERREIRA, A.B. H. Novo dicionário Aurélio. Rio de Janeiro: Ed. Nova Fronteira, 1993.

ISHIKAWA, K. Controle de Qualidade Total: à maneira japonesa. Rio de Janeiro: Ed. Campus, 1993.

PURI, S. C. ISO 9000. Certificação-Gestäo da Qualidade Total. Rio de Janeiro: Qualitymark, 1994.

RAMOS, M. L. T et all. Implantação dos Indicadores de Qualidade no serviço de Enfermagem do Hospital Israelita Albert Einstein. In: ENFTEC, 4., 1994, São Paulo. Anais... Säo Paulo, 1994.

VAZ, M. J. R.; BARROS, S. M. O. Enfermeiros e qualidade: alguns conceitos. Acta Paul. Enf., São Paulo, v.10, n. 3, p. 7-13, 1997.

ASSOCIAÇÃO BRASILEIRA DE NORMAS TÉCNICAS. NBR ISO 9002. Sistemas de Qualidade Modelo para garantia da qualidade em produção, instalação e serviços associados. Rio de Janeiro: ABNT dez. 1994.

Recebido em outubro de 1998

Aprovado em agosto de 1999 